\title{
NOUVELLE
}

\section{Le venin de mamba vert, espoir thérapeutique dans la polykystose rénale}

Cyprien Jahan ${ }^{1}$, Philippe Robin ${ }^{2}$

\author{
${ }^{1}$ Ml Biologie Santé, ENS Paris Saclay, 94230 Cachan ; \\ ${ }^{2}$ Institut des sciences du vivant Frédéric Joliot, CEA Saclay, \\ 91190 Gif sur Yvette, France. \\ cyprien.jahan@ens-paris-saclay.fr \\ philippe.robin@cea.fr
}

La maladie polykystique rénale autosomique dominante

Les maladies polykystiques rénales (acronyme anglais PKD) sont des maladies génétiques potentiellement mortelles dues à la présence dans le rein de nombreux kystes remplis de fluide. Ces kystes empêchent le bon fonctionnement de l'organe et peuvent entrainer à terme une insuffisance rénale [1]. La PKD autosomique dominante $(A D P K D)^{1}$ est une des maladies monogéniques les plus communes dans le monde; sa prévalence est de 1/1000 en France [2, 3]. Malgré de nombreux essais, aucun traitement pleinement satisfaisant n'a encore été développé, mais une avancée clé a été l'observation d'une accumulation anormale d'AMPc (adénosine monophosphate cyclique) dans les reins affectés. L'AMPc, synthétisé par l'adénylyl cyclase, une enzyme dont l'activité est régulée par certaines protéines $G$ hétérotrimériques, provoque l'accumulation de fluide dans le lumen et stimule la prolifération cellulaire par activation de la voie des MAPK (mitogen activated protein kinase) de la famille ERK (extracellular signal regulated kinase) [4]. Ces protéines $G$ hétérotrimériques sont elles-mêmes régulées par des protéines de la famille des récepteurs couplés aux protéines $G$ que l'on trouve à la membrane cellulaire. De précédentes études ont repéré qu'un membre de cette famille - le récepteur

\footnotetext{
${ }^{1}$ Deux gènes peuvent être mutés dans la PKD autosomique dominante. Dans $85 \%$ des cas il s'agit de PKD1, dans $15 \%$ des cas de $P K D 2$. PKD1 est un gène localisé en 16p13, qui code une protéine membranaire, la polycystine-1 (PC-1), un possible mécanorécepteur dont la fonction précise est inconnue. Le gène PKD2 sur le chromosome 4 code la polycystine-2 $(P C-2)$, un canal calcique de la famille TRP.
}

de la vasopressine de type $2(\mathrm{~V} 2 \mathrm{R})^{2}$ est présent en grande quantité dans les zones de développement de kystes. Ce récepteur apparait donc comme une cible thérapeutique potentielle étant donné qu'il est couplé à la protéine $G_{s}$, activatrice de l'adénylyl cyclase. Ainsi, une molécule capable de bloquer ce récepteur V2 permettrait de réduire le niveau d'activation de l'adénylyl cyclase et de ralentir le développement de la maladie $[5,6]$. Le tolvaptan, un antagoniste du récepteur $V 2 R$, est déjà utilisé pour traiter I'ADPKD, et ce, avec une certaine efficacité. Cependant, il a comme inconvénients d'être peu sélectif, bloquant d'autres récepteurs parmi lesquels le récepteur VI de la vasopressine, et d'induire une toxicité hépatique [5]. Une alternative aux «petites molécules » thérapeutiques pourrait être représentée par les peptides présents dans le venin des animaux venimeux. En effet, un nombre important de ces peptides naturels sont des antagonistes de récepteurs et ils ont des propriétés intéressantes telles qu'une haute affinité et une grande sélectivité vis-à-vis de leur récepteur cible [7]. Par le passé, les travaux menés dans le service d'ingénierie des protéines du CEA à Saclay ont conduit à l'identification et la caractérisation de toxines peptidiques ciblant des récepteurs couplés aux protéines $G$ (dont fait partie le V2R) à partir du venin des serpents de la famille des mambas

\footnotetext{
${ }^{2}$ La vasopressine (AVP) ou hormone antidiurétique (ADH) est un peptide de 9 acides aminés, synthétisé par les neurones de l'hypothalamus. La vasopressine est sécrétée dans la circulation périphérique et exerce ses fonctions via deux types de récepteurs: V2 dans le rein (action antidiurétique), et V1 assez ubiquitaires (action de contraction vasculaire notamment).
}

[8]. Dans ce contexte, l'objectif des travaux menés par Nicolas Gilles et ses collaborateurs a été de chercher à identifier et à caractériser, in vitro et in vivo, des peptides issus du venin de mamba vert qui pourraient bloquer spécifiquement le récepteur V2R [9]. À terme, un tel peptide pourrait s'avérer un outil thérapeutique contre la PKD.

\section{Identification et purification}

de la mambaquarétine- 1

L'équipe a tout d'abord cherché à identifier des molécules potentiellement actives contre le V2R dans le venin de mamba vert. Pour cela, les centaines de molécules présentes dans le venin ont été séparées les unes des autres par des techniques de chromatographie liquide, puis testées pour leur capacité à bloquer le récepteur V2R, c'est-à-dire à empêcher son ligand naturel, la vasopressine, de s'y fixer. Un tel peptide a été identifié, cette toxine a été appelée mambaquarétine-1. Sa séquence a été déterminée, elle comprend 57 acides aminés et 3 ponts disulfure, et l'équipe est parvenue à synthétiser le peptide chimiquement. La mambaquarétine-1 agit exclusivement sur V2R (150 autres récepteurs ont été testés), sur lequel elle se fixe avec une haute affinité ( $\mathrm{Ki}=$ 2,8 nM). La mambaquarétine-1 possède donc les qualités recherchées de haute affinité et haute sélectivité pour le V2R.

La mambaquarétine- 1 bloque l'activation des voies de signalisation induites par le V2R

Les chercheurs se sont alors intéressés aux effets de la mambaquarétine-1 


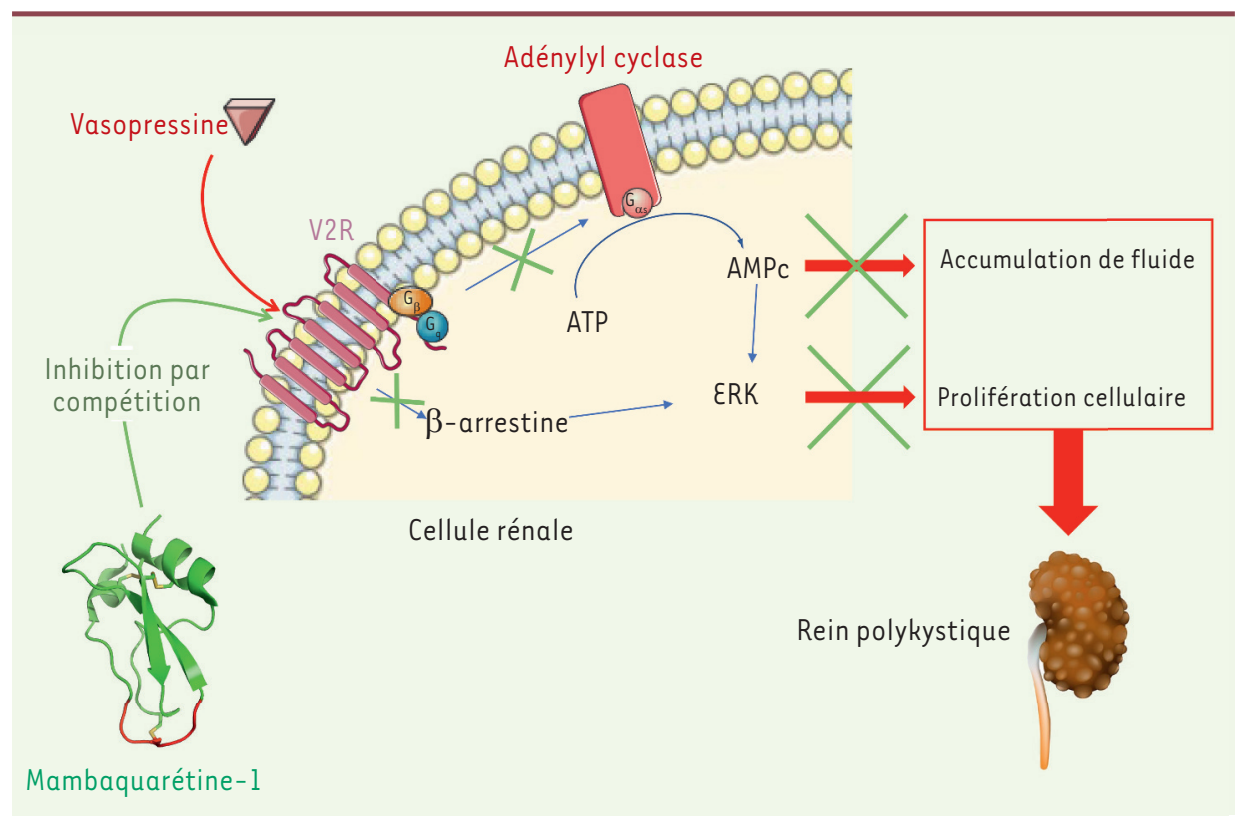

Figure 1. Polykystose rénale : mécanisme pathologique et action de la mambaquarétine-1. En absence de mambaquarétine-1, la vasopressine active son récepteur $V 2 R$ et les voies en aval qui favorisent le développement de la maladie (flèches rouges). En présence de mambaquarétine-1, qui se fixe au récepteur $V 2 R$ et empêche la vasopressine de s'y lier, les voies de signalisation ne sont plus activées (croix vertes), ce qui permet de protéger le rein. La partie rouge de la structure de la mambaquarétine- 1 correspond à la zone d'interaction avec le récepteur V2R.

sur les voies de signalisation régulées par V2R. Celles-ci font intervenir trois acteurs principaux: l'adénylyl cyclase, qui conduit à la production d'AMPc; la $\beta$-arrestine, qui joue un rôle dans la désensibilisation du récepteur; et les MAPK ERK, dont l'activation dépend des deux premiers et qui jouent un rôle essentiel dans la prolifération cellulaire. Les résultats obtenus sur des cellules de hamster génétiquement modifiées pour surexprimer le récepteur V2R humain, montrent que la mambaquarétine- 1 n'active pas ces voies mais que, au contraire, elle inhibe leur activation par la vasopressine. La mambaquarétine-1 joue un rôle d'antagoniste pour chacune de ces voies de signalisation, et son action est purement compétitrice visà-vis de la vasopressine. Le même effet antagoniste a été obtenu lorsque des expériences comparables ont été réalisées dans des cellules rénales immortalisées exprimant constitutivement $V 2 R$, qui sont plus proches des cellules kystiques.

La mambaquarétine- 1 réduit le nombre de kystes et améliore la fonction rénale dans un modèle murin d'ADPKD

Afin d'avoir un aperçu des effets in vivo de la mambaquarétine-1 sur les reins normaux et polykystiques dans la perspective de sa potentielle utilisation thérapeutique, les chercheurs ont utilisé des souris homozygotes pcy (mutation spontanée dans le gène NPHP3 [nephronophthisis 3] impliqué dans la différenciation des cellules rénales), dont le phénotype est proche de celui des patients atteints d'ADPKD. L'administration par voie intrapéritonéale de mambaquarétine-l a un effet aquarétique, c'est-à-dire qu'elle provoque une augmentation du volume de l'urine ainsi qu'une diminution de son osmolarité. In vivo, la mambaquarétine-l inhibe donc l'effet antidiurétique de la vasopressine. Il est important de noter qu'il n'y a pas d'augmentation dans l'urine de la concentration des électrolytes et des protéines - donc pas de fuite urinaire excessive de ces éléments - et on n'observe aucun effet néfaste pour la souris au niveau des différents organes. Lorsque l'on compare les souris pcy ayant reçu de la mambaquarétine-1 aux souris pcy non traitées, on observe une diminution du nombre de kystes ainsi qu'une amélioration fonctionnelle rénale dont témoigne une normalisation des taux d'urée et de créatinine dans le sang.

\section{Conclusion}

Cette étude a permis d'identifier la mambaquarétine-1, une toxine contenue dans le venin du mamba vert, dont la morsure peut être mortelle pour l'homme, comme un peptide potentiellement efficace dans la polykystose rénale. En effet, en se fixant de manière spécifique au récepteur de la vasopressine V2R de type 2, elle agit comme un antagoniste compétitif de $V 2 R$, inhibant en aval les voies de signalisation AMPc, $\beta$-arrestine et MAPK ERK. La diminution de la quantité d'AMPc permet notamment de réduire l'accumulation de fluide dans le lumen et contribue à diminuer la prolifération cellulaire, et, en conséquence, le nombre de kystes, ce qui contribue à l'amélioration de la fonction des reins polykystiques. Cependant, cette étude ayant été réalisée dans un modèle murin, il faudra en confirmer les résultats dans d'autres modèles avant d'envisager l'utilisation de la mambaquarétine-1 chez l'homme. Quoi qu'il en soit, l'identification d'une perspective thérapeutique prometteuse dans l'ADPKD illustre la formidable ressource en molécules bioactives que constituent les toxines animales et invite à renforcer la recherche dans ce domaine. $\diamond$

Green mamba venom, a therapeutic promise in polycystic kidney disease 


\section{RÉFÉRENCES}

1. Sun Y, Zhou H, Yang Bx. Drug discovery for polycystic kidney disease. Acta Pharmacol 2011 ; 32 : 805-16.

2. Willey CJ, Blais JD, Hall AK, et al. Prevalence of autosomal dominant polycystic disease in the European Union. Nephrol Dial Transplant 2016 ; 32 : 1356-63.

3. Torres VE, Harris PC. Autosomal dominant polycstic disease: The last three years. Kidney Int $2009 ; 76$ : 149-68.

4. Mangoo-Karim R, Uchic ME, Grant M, et al. Renal epithelial fluid secretion and cyst growth: The role of cyclic AMP. FASEB J $1989 ; 3: 2629-32$.
5. Torres VE, Chapman AB, Devuyst 0 , et al. Tolvaptan in patients with autosomal dominant polycystic kidney disease. N Engl J Med 2012 ; 367 : 2407-18.

6. Gattone VH, Wang X, Harris PC, Torres VE. Inhibition of renal cystic disease development and progression by a vasopressin V2 receptor antagonist. Nat Med 2003; $9: 1323-6$.

7. King GF, ed. Venoms to drugs, drug discovery. Royal society of Chemistry, London 2015; P001-320.

8. Rouget C, Quinton L, Maïga A, et al. Identification of a novel snake peptide toxin displaying high affinity and antagonist behaviour for the $\alpha 2$-adrenoceptors. $\mathrm{Br} J$ Pharmacol 2010 ; 161 : 1361-74.
9. Ciolek J, Reinfrank H, Quinton L, et al. Green mamba peptide targets type-2 vasopressin. Proc Natl Acad Sci USA 2017 ; 114 : 7154-9.

10. Gasparini S, Danse JM, Lecoq A, et al. Delineation of the functional site of $\alpha$-dendrotoxin: The functional topographies of dendrotoxins are different but share a conserved core with those of other Kvl potassium channel-blocking toxins. J Biol Chem $1998 ; 273$ : 25393-403.

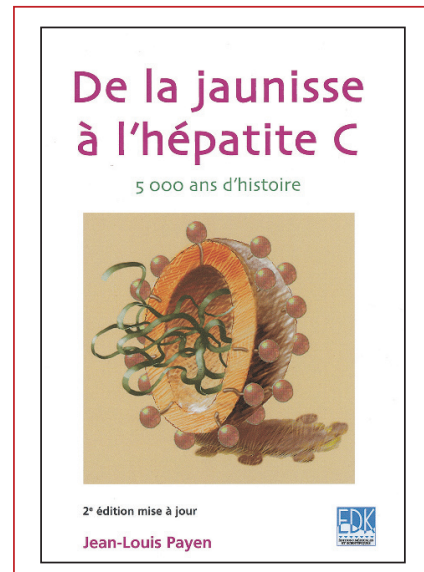

ISBN : 978-2-8425-4136-1 128 pages a jaunisse est un symptôme facilement identifiable; il paraissait bien naturel que l'homme, confronté à
une modification de la couleur de ses yeux et de sa peau ait de tous temps recherché les causes de cette
transformation. II n’est donc pas surprenant que le premier traité de médecine, écrit 3000 ans avant J.C. par un médecin sumérien, décrive déjà la jaunisse. À chaque époque de l'histoire de la médecine, les praticiens, influencés par les concepts médicaux de leur temps, attribuèrent une ou plusieurs explications particulières à ce symptôme. Ainsi, du démon Ahhâzu des Sumériens à la sophistication des biotechnologies qui permirent la découverte du virus de l'hépatite $C$, le lecteur cheminera sur une période de 5000 ans au travers des différents continents.

Ici encore, l'histoire se révèle une formidable source de réflexion : le foie souvent impliqué dans l'apparition des jaunisses est-il le siège de l'âme?

Les expérimentations humaines chez des volontaires ou chez des enfants handicapés mentaux étaient-elles justifiées pour permettre la découverte des virus des hépatites?

Le formidable développement de la transfusion sanguine, des vaccinations, mais aussi de la toxicomanie explique-t-il les épidémies d'hépatites du xxe siècle?

Autant de questions qui sont abordées dans ce livre passionnant et accessible à tous.

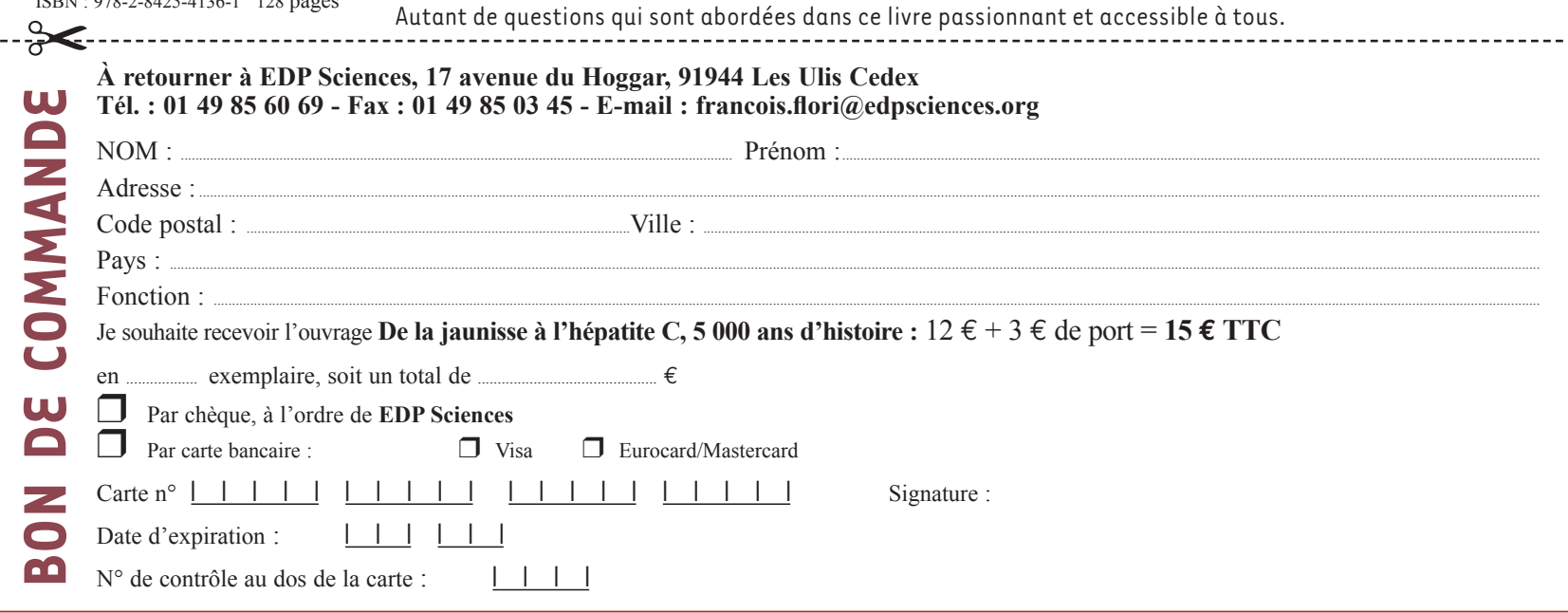

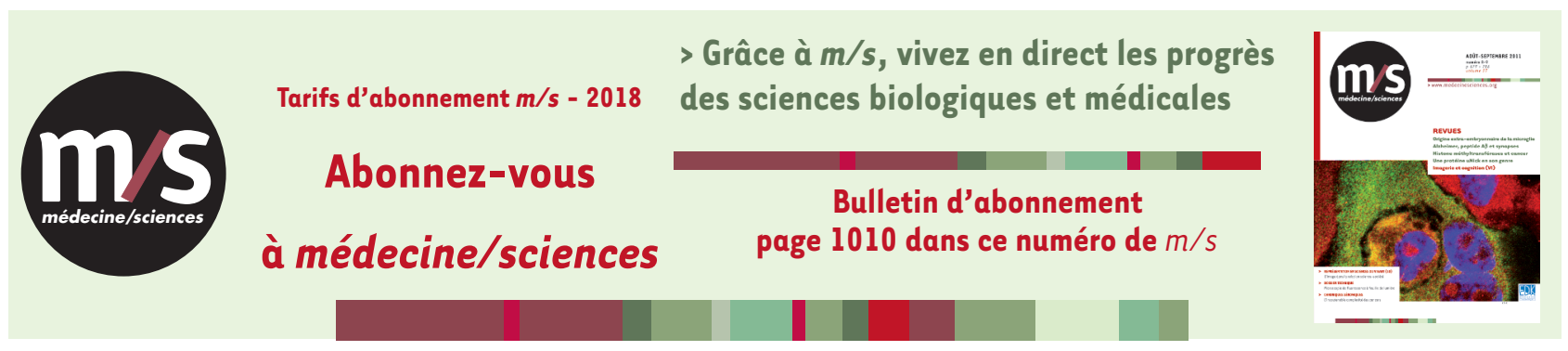

